\title{
RELATIVITY INDEX OF THE RELIABILITY OF SPACE SYSTEMS
}

\author{
Nikolay Petrov \\ Technical University of Sofia, \\ Kliment Ohridski 8, Sofia-1000, Bulgaria, \\ E-mail: nikipetrov_1953@abv.bg
}

\begin{abstract}
According to the special theory of relativity by Einstein, it can be affirmed that two events coincide in space only when objects are shown (or system of objects); according to latter the position of these events is defining. In this way the term "position in space" is relative too. When we talk about the position of one object in space - it's actually its position among other objects. However, if we have to give answer to the question "where is the current object?" without mentioning about the condition of adjacent objects, it follows that this question will be meaningless. In this article has been done a trial for taking of theoretical dependence, which defines the probability for no-failure of mobile space systems (spacecraft), moving with determinate speed towards an observer on the Earth.
\end{abstract}

Keywords: special theory of relativity; reliability.

\section{INTRODUCTION}

In the literature sources [1-8] is defined the main law of reliability for technical systems (TS) which are at earth conditions and moving with low speed (in comparison with the speed of light). So, towards static observer on planet Earth it is possible the defining of the following equation, which is determining the probability for no-failure $P_{N F}(\Delta t)$ of TS in interval of observation $\left[t_{1}, t_{2}\right]$.

$$
P_{N F}\left(t_{1}, t_{2}\right)=e^{-\int_{t_{1}}^{t_{2}} \omega(t) d t}
$$

where $\omega(t)$ is the intensity of the failures in the observed period of time $\left[t_{1}, t_{2}\right]$.

It follows that the valuation of the intensity of the failures $\hat{\omega}(t)$ of TS for the period of time $\Delta t$ is defined by next formula [2, 11 and 14]

$$
\hat{\omega}=\frac{\sum_{i=1}^{n} r_{i}\left(t_{1}, t_{2}\right)}{n\left(t_{1}, t_{2}\right) \sum_{i=1}^{n} \tau_{i}\left(t_{1}, t_{2}\right)},
$$

where $r_{i}\left(t_{1}, t_{2}\right)$ is the number of the failures of the $i$-th observed TS in the interval $\Delta t=t_{2}-t_{1}$; $n=n\left(t_{1}, t_{2}\right)-$ number of the observed TS in $\Delta t$; $\tau_{i}\left(t_{1}, t_{2}\right)$ - time for no-failure work of the $i-$ th observed TS in the interval $\Delta t$.

\section{EXPOSITION}

If we proceed from the assumption for the special theory of relativity of Albert Einstein and suppose that the period of time $\Delta t$ for static observer who is on planet Earth, is defined by [9, 10] then

$$
\Delta t=\frac{\Delta t_{M S}}{\sqrt{1-\frac{V_{M S}^{2}}{c^{2}}}},
$$

where $\Delta t_{M S}$ is period of time for mobile system (spacecraft) which is moving with speed $V_{M S} ; c-$ the speed of light in vacuum accepted from Einstein for constant with value $300000 \mathrm{~km} / \mathrm{s}$.

As these conditions it can be done mathematic analysis of the problem in the context of theory of relativity by the following way.

In equation (3) we have

$$
V_{M S}=c \sqrt{1-\left(\frac{\Delta t_{M S}}{\Delta t}\right)^{2}} .
$$

In (4) for the speed of the mobile system (spacecraft) towards planet Earth, is introduced the 
concept - relativity index of reliability $R\left(V_{M S}\right)$ which is defined by the equation:

$$
R\left(V_{M S}\right)=\Delta t_{M S} / \Delta t
$$

From (5) it follows that the period of time $\left(t_{1}, t_{2}\right)$ of the mobile system in which is defined the intensity of the failures $\omega_{M S}(t)$ and the probability for no- failure $P_{N F M S}(t)$ is:

$$
\Delta t_{M S}=t_{2}-t_{1}=R\left(V_{M S}\right) \Delta t
$$

From (6) with assumption for ordinary and lack of consequences of the failures of mobile system, for its intensity $\omega_{M S}(t)$ are the following equations:

$$
\begin{aligned}
& \omega_{M S}(t) / \omega(t)=\Delta t_{M S} / \Delta t, \\
& \omega_{M S}(t)=R\left(V_{M S}\right) \omega(t) .
\end{aligned}
$$

Therefore for the mobile system (spacecraft, moving with high speed) towards the planet Earth, the main reliability's law $P_{N F M S}(t)$ (from observer on planet Earth point of view) will be:

$$
P_{N F M S}\left(t_{1}, t_{2}\right)=e^{-\int_{t_{1}}^{t_{2}} \omega_{M S}(t) d t}
$$

The analysis of equation (9) shows that for mobile material system observed from planet Earth and moving with speed $V_{M S} \geq c .10^{-5}=3 \mathrm{~km} / \mathrm{s}$ [15] (the rockets from US anti-missile defense have approximate speed $6,6 \mathrm{~km} / \mathrm{s}$ ) the main law of reliability will be:

$$
P_{\text {NFMS }}\left(t_{1}, t_{2}\right)=e^{-R\left(V_{M S}\right) \int_{t_{1}}^{t_{2}} \omega(t) d t}
$$

where $V_{M S}$ is speed of mobile material system, moving towards planet Earth on which is evaluating the reliability.

On its part the relativity index of reliability $R\left(V_{M S}\right)$ is defined by (7) as:

$$
R\left(V_{M S}\right)=\frac{\omega_{M S}(t)}{\omega(t)}=\frac{\Delta t_{M S}}{\Delta t}=f\left(V_{M S}\right),
$$

where $f\left(V_{M S}\right)=a_{n} V_{M S}^{n}+a_{n-1} V_{M S}^{n-1}+\ldots \ldots+a_{1} V_{M S}+a_{0}$.
The function defines the modification of the speed of the mobile systems $f\left(V_{M S}\right)$ is a function which defines the modification of the speed of the spacecraft $V_{M S}$ towards planet Earth and it is approximating in meaning of the least squares $[12,13]$.

As a condition that spacecraft is shipped around planet Earth (for example the space shuttle "Atlantis") and is made the analysis of the intensity of the failures of the communication system at Earth conditions (immobile system) and at space conditions (mobile system) then (10) is in the form

$P_{N F M S}\left(t_{1}, t_{2}\right)=\left\{e^{-\int_{t_{1}}^{t_{2}} \omega(t) d t}\right\}^{R\left(V_{M S}\right)}=\left\{P_{N F S E C}\left(t_{1}, t_{2}\right)\right\}^{R\left(V_{M S}\right)}(12)$.

In (12) $P_{N F S E C}\left(t_{1}, t_{2}\right)$ is the reliability (the probability for no-failure) of investigated communication system at Earth conditions (SEC).

From (12) it follows that in the space conditions will raise the probability of the observed communication system (and by analogy for another technical systems). For examples can show known accidents with spacecrafts. They are coming exceptional after the start from the planet Earth and passing through the atmosphere. Then there exist and another factors of the reliability $[14,15]$.

\section{EXAMPLE FOR THE RELIABILITY INVESTIGATION}

Let we observed 10 identical communication systems to the space shuttle "Atlantis" in continuation of 1000 hours continuous work (for the begin in Earth conditions and after this in a space conditions). The common results of the failures at Earth conditions are 3 and in space conditions is 1 .

From (1) and (11) it follows that for the intensity of the failures of the system at Earth conditions (SEC) and in space conditions (SSC) we have

$$
\begin{aligned}
& \hat{\varpi}_{S E C}\left(t_{1}, t_{2}\right)=\frac{3}{10.1000}=3 \cdot 10^{-4} \mathrm{fit} ; \\
& \hat{\varpi}_{S S C}\left(t_{1}, t_{2}\right)=\frac{3}{10.1000}=1 \cdot 10^{-4} \mathrm{fit} ; \\
& R(V)=\frac{\hat{\varpi}_{S S C}\left(t_{1}, t_{2}\right)}{\hat{\varpi}_{S E C}\left(t_{1}, t_{2}\right)}=\frac{1.10^{-4}}{3 \cdot 10^{-4}}=0,33,
\end{aligned}
$$


where fit is the unit for the intensity of the failures equivalent to 1 failure for 1 hour.

It follows the calculations about the probability of the system at earth conditions (SEC) with approximate formula for stationary, ordinary and without consecutive the failures $[1,14,15]$.

$$
P_{N F S E C}\left(t_{1}, t_{2}\right)=e^{-\omega_{S E C}\left(t_{1}, t_{2}\right) \tau_{\Sigma}}=e^{-0,3 \cdot 10^{-3} \cdot 10^{3}}=0,742 .
$$

From (12) it follows that the probability for nofailure of investigated communication system at space conditions (SSC) will be

$$
P_{N F S S C}\left(t_{1}, t_{2}\right)=\left\{P_{N F S E C}\left(t_{1}, t_{2}\right)\right\}^{R\left(V_{M S}\right)}=0,742^{0,33}=0,906
$$

After a check for the indefinite of calculations as used classical calculate (1) for the reliability of communication system of "Atlantis" in the space conditions we have

$$
P_{N F S S C, 1}\left(t_{1}, t_{2}\right)=e^{-\omega_{S S C}\left(t_{1}, t_{2}\right) \tau_{\Sigma}}=e^{-0,1 \cdot 10^{-3} \cdot 10^{3}}=0,905 .
$$

Consequently the absolute indefinite of calculations for the reliability of communication system in the space conditions is

$$
\Delta P_{N F S S C}\left(t_{1}, t_{2}\right)=0,906-0,905=0,001 .
$$

The decision we had made are in condition that the speed of light in vacuum is a constant, i.e. the spacecraft is moving out of the earth atmosphere.

\section{CONCLUDING REMARKS}

From this reliability analysis in the context of the special and common theory of relativity we can make these conclusions:

It is necessary the introduction of relativity index in the main law of reliability for system from planet Earth and moving forward to it with high speed material object. The relativity index of reliability can be connected only to changes in conditions in which are the observed object; these conditions are engendered in result of different by kind interactions with the objective reality.

\section{REFERENCES}

[1] Gindev E. Introduction in the theory and the practice of reliability. Part 2. Bases of the practical reliability. Sofia, Academic publishers "Prof. Marin Drinov", 2000.

[2] Gindev E. Introduction in the theory and the practice of reliability. Part 2. Bases of the practical reliability. Sofia, Academic publishers "Prof. Marin Drinov", 2002.

[3] Gnedenko B., U. Beliaew, A. Solaviow. Mathematical methods in the reliability theory. Moscow, Publ. "Science", 1965.

[4] Avizienis A. and L. Lapr. Dependability Basic Concept and Terminology. Springer Verlag, Wiena, New York, 1999.

[5] O'Connor, P. Practical. Reliability Engineering. John Willey and Sons, Copyright, 1997.

[6] Page L. Probability for Engineering with Applications to Reliability. Paris, Computer Science Press, Inc., 1999.

[7] Smith D. Reliability and Maintainability in Perspective. London, Macmilan Publishers, 1995.

[8] Tobias P., D. Trindade. Applied Reliability. New York, Van Nostrad Reinhold Company, 1996.

[9] Einstein A. Special and common theory of the relativity. Sofia, Pub. "Science and art", 1965.

[10] Einstein A. Work about theory of the relativity (1905-1920). Moscow, Complete science works. Publ., "Science", 1965.

[11] Walpole R., R. Myers. Probability and Statistics for Engineers and Scientist. New York, Macmilan Publishing Company, 1995.

[12] Sendov B1., V. Popov. Numerical methods. Sofia, University publisher "St. Kliment Ohridski", 1996.

[13] Bahvalov N., N. Jidkov, G. Kobelnikov. Numerical methods. Moscow, Publ. "Binomial", 2006.

[14] Petrov N. Exploitation reliability of risk technical systems. Burgas, "Prof. Asen Zlatarov" University, Publ. "Utshkov", 2002.

[15] Petrov N. Reliability analyze in context of the special theory of the relativity. Ruse University, Bulgaria, 2007. pp. 57-61.

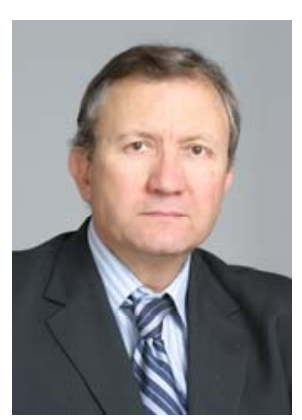

Nikolay Ivanov Petrov received $M$. Sc. Degree from the National University "V. Levski", Aviation Faculty, specialty "Radio Equipping of Aircrafts". He got Ph.D. with Doctorate Thesis "Optimizing and Control of the Technical Usage of Air Systems". And he has defended Dissertation by Automated Systems for Information Technology and Management in the Institute for Perspective Defense Research - Sofia, Bulgaria. Since 2001 he has been working as an Assistant Professor and Associate Professor at the University "Professor D$r$ Assen Zlatarov", Burgas (Bulgaria) and Trakia University, Stara Zagora, Yambol (Bulgaria). In 
2004 he graduated UNWE-Sofia, subject "Economics of the Safety".

His research activities are centered on Automated Systems, Reliability and Risk of Technical Systems and Electronic Devices for Measuring.

In 2007 he was awarded Professor of the "Trakia University" by specialty, Atomized Systems for Treatment of Information and Control. He has more than 300 scientific works, publications and developments, 50 of which - abroad. He has published 25 scientific books and textbooks, 5 of which - monographs.

On 29 September 2009 he is chosen for Professor in Technical University, Sofia.

Professor Petrov has been taking part in many scientific congresses and conferences, for example in Vienna (Austria), Athens (Greece), Brno (Czech), Lvov (Ukraine), Istanbul (Turkey) and others. 